Reprod. Nutr. Dévelop., 1985, 25 (1 B), 215-216.

\title{
Régénération du tissu adipeux après lipectomie chez des lapins âgés de 6 et 12 mois
}

\author{
J. NOUGUĖS, Y. REYNE, A. VÉZINHET
}

avec la collaboration technique de Odette FRECCERO. Thérèse CHERY, Josette SORIANO et E. BOUTHIER

Station de Physiologie animale, I.N.R.A.,

E.N.S.A., Place Viala, 34060 Montpellier Cedex, France.

Une série d'expériences a été réalisée pour étudier les effets d'une ablation chirurgicale du tissu adipeux chez le lapin. Des lapins mâles Néozélandais ont été lipectomisés ou pseudo-opérés à 6 ou 12 mois, âges auxquels, respectivement, la taille et le nombre d'adipocytes se stabilisent chez cette espèce (Nouguès et Vézinhet, 1977). Quatre-vingt pour cent environ des tissus adipeux périrénal et omental et la totalité des tissus interscapulaire et inguinal ont été prélevés. L'ensemble des tissus enlevés représentait en moyenne $35 \%$ de la masse adipeuse totale chez les animaux opérés à l'âge de 6 mois et $48 \%$ chez les animaux opérés à l'âge de 12 mois. Tous les lapins ont été sacrifiés 3 mois après lipectomie et soigneusement disséqués. La cellularité des tissus adipeux périrénal et interscapulaire a été déterminée au moment de l'opération et au moment du sacrifice pour les tissus se régénérant. Afin de détecter une éventuelle compensation sur la taille des cellules des tissus non opérés, la taille des adipocytes a également été mesurée pour les tissus adipeux mésentérique et cervical par fixation des cellules au tetroxyde d'osmium selon la méthode de Hirsch et Gallian (1968).

Trois mois après lipectomie, nous n'avons pas observé de régénération des tissus interscapulaire, inguinal et omental alors qu'il y a une régénération importante du tissu périrénal quel que soit l'âge des animaux au moment de l'opération (Reyne, Nouguès et Vézinhet, 1983). Les tissus adipeux non opérés n'ont pas montré de croissance compensatrice. La régénération du tissu adipeux périrénal est due à une hyperplasie cellulaire et se produit approximativement à la même vitesse chez les animaux adultes et chez les animaux âgés de 6 mois. Par ailleurs, nous n'avons pas observé de différences significatives entre les animaux lipectomisés et les pseudo-opérés dans leur consommation d'aliment ou leur évolution pondérale après opération.

L'absence de régénération des tissus adipeux sous-cutanés (interscapulaire et inguinal) pouvait résulter simplement d'une réponse plus lente de ces tissus après lipectomie ou de la destruction totale de la trame neurovasculaire essentielle au métabolisme lipidique. Des travaux complémentaires nous ont permis d'exclure ces deux hypothèses. En effet, nous n'avons pas noté de régénération du tissu

Reproduction Nutrition Développement, $n^{\circ} 1$ B-1985. -5 
adipeux interscapulaire 6 mois après opération ou lorsqu'on effectue seulement une lipectomie partielle de ce tissu. II y a donc bien des différences entre tissus dans leurs aptitudes à se régénérer.

L'ensemble des résultats obtenus, suggérant que la régénération du tissu adipeux chez le lapin dépend de la localisation anatomique, est en accord avec les travaux réalisés chez le rat par d'autres équipes (Larson et Anderson, 1978; Faust, Johnson et Hirsch, 1979, etc...). Les différences de comportement entre tissus adipeux de diverses localisations anatomiques pourraient être le reflet de différences dans les caractéristiques intrinsèques aux cellules précurseurs des adipocytes et/ou de différences dans l'environnement local des cellules.

$10^{e}$ Réunion du groupe Développement I.N.R.A., Rennes, 9-10 mai 1984.

\section{Références}

FAUST I. M., JOHNSON P. R., HIRSCH J., 1979. Adipose tissue regeneration in adult rats. Proc. Soc. exp. Biol. Med., 161, 111-114.

HIRSCH J., GALLIAN E., 1968. Methods for the determination of adipose cell size in man and animals. J. Lipid Res., 9, 110-119.

LARSON K. A., ANDERSON D. B., 1978. The effects of lipectomy on remaining adipose tissue depots in the Sprague-Dawley rat. Growth, 42, 469-477.

NOUGUĖS J., VEZINHET A., 1977. Evolution pendant la croissance de la cellularité du tissu adipeux chez le lapin et l'agneau. Ann. Biol. anim. Biochim. Biophys., 17, 799-806.

REYNE Y., NOUGUĖS J., VEZZINHET A., 1983. Adipose tissue regeneration in 6-month old and adult rabbits following lipectomy. Proc. Soc. exp. Biol. Med., 174, 258-264. 